邓子辰 ${ }^{(12 *}$, 曹珊珊 ${ }^{(1)}$, 李庆军 ${ }^{(1)}$, 蒋宪宏 ${ }^{1}$

(1) 西北工业大学力学与土木建筑学院, 西安 710072;

(2) 西北工业大学理学院, 西安 710072

*E-mail: dweifan@nwpu.edu.cn

收稿日期: 2016-05-02; 接受日期: 2016-08-31; 网络出版日期: 2016-11-24

国家自然科学基金(批准号: 11432010)和西北工业大学研究生创意创新种子基金(编号: Z2016093)资助项目

\begin{abstract}
摘要空间太阳能发电作为获取清洁、可再生的能源的重要途径, 受到了社会的广泛关注. 本文基于 Hamilton 体系研究了太阳帆塔式空间太阳能电站在轨运行的动力学与系统的稳定问题. 通过建立太阳帆塔简化结构模型 和引入广义坐标以及广义动量, 根据变分原理, 得到 Hamilton 体系下太阳帆塔系统在轨运行的动力学控制方程; 采用辛 Runge-Kutta 方法进行求解分析, 并与传统的四阶 Runge-Kutta 方法对比, 数值结果表明了辛 Runge-Kutta 方法能够保持系统的能量和系绳弹性振动的特性; 最后, 根据力学平衡原理得到了系统的平衡点, 并通过数值 算例研究了系统在平衡点附近的稳定性.
\end{abstract}

关键词空间太阳能电站, Hamilton 系统, 辛 Runge-Kutta 方法, 太阳帆塔

\section{1 引言}

伴随着与日俱增的能源需求及环境安全问题, 科学家开始设想将太阳能作为未来发展的主要资源. 1968 年, 美国的 Glaser 博士首次提出建设空间太阳 能电站的构想 ${ }^{[1]}$. 随后, 以美国和日本为首的发达国 家展开了一系列的探索论证工作, 到目前为止, 国际 上已经提出了几十种空间太阳能电站设计构想 ${ }^{[2]}$.

欧洲于 1998 年实施了“空间探索和利用的系统 概念、结构和技术”计划, 提出了太阳帆塔概念. 太阳 帆塔式空间太阳能电站采用了轻型结构一太阳帆,
大大减轻了结构的总质量, 同时降低了装配难度, 具 有广阔的应用前景 ${ }^{[2 \sim 4]}$. 太阳帆塔式空间太阳能电站 作为未来的一种可再生能源系统, 是一项宏伟的空 间和地面工程, 涉及到许多重要的技术领域 ${ }^{[5]}$, 而太 阳帆塔的动力学与控制是其中至关重要的一环. 太 阳帆塔的动力学与控制直接影响着太阳帆板的对日 指向、发射天线的对地指向以及太阳帆塔系统的轨道 寿命, 而由于太阳帆塔结构的复杂, 很难对其在轨运 行动力学与控制过程进行地面实验研究, 因此, 太阳 帆塔动力学建模和数值仿真具有极其重要的作用. 目前, 国际上对于大型航天器在轨运行的动力学与

引用格式: 邓子辰, 曹珊珊, 李庆军, 等. 基于辛 Runge-Kutta 方法的太阳帆塔动力学特性研究. 中国科学: 技术科学, 2016, 46: 1242-1253 Deng Z C, Cao S S, Li Q J, et al. Dynamic behavior of sail tower SPS based on the symplectic Runge-Kutta method (in Chinese). Sci Sin Tech, 2016, 46: 1242-1253, doi: 10.1360/N092016-00163 
控制问题已经有了大量的研究, 诸如绳系卫星系 统 $^{\left[{ }^{6]}\right.}$ 、空间交会卫星系统 ${ }^{[7]}$ 、自旋展开太阳帆系统 ${ }^{\left[{ }^{[}\right]}$ 等, 然而面对太阳帆塔这一类复杂结构, 其动力学与 控制问题显得尤为突出. 太阳帆塔动力学与控制问 题与传统的卫星不同, 需要建立新的动力学模型来 研究它的动力学特性.

太阳帆塔系统动力学与控制属于一种比较复杂 的非线性动力学系统. 此类非线性系统广泛存在于 工程实际中, 由于其动力学方程中存在复杂的非线 性项, 一般得不到解析解, 因此, 很多学者逐渐将科 研重点放在研究这些复杂动力学方程的数值解上. 而对于保守系统的动力学问题, 由于 Hamilton 系统 有很多优越的内在特性, 如它的相流形能保持相空 间的面积和体积不变, 保持系统的能量和动量等, 因 而在科学研究与工程实践中都得到了广泛的应用 ${ }^{[9]}$. 然而用一般的数值方法对系统仿真时, 这些性质往 往得不到保持, 因此, Hamilton 体系的数值方法研究 近来一直是力学界和计算数学界的重要课题 ${ }^{[9,10]}$, 而 这些研究的核心问题便是系统在数值计算中实现“保 辛, ${ }^{[11]}$. 保辛思想的首次提出, 可追溯到 1984 年在北 京召开的第五届国际双微会议上, 冯康院士首次提 出了基于辛几何原理计算 Hamilton 系统的辛方法 ${ }^{[12]}$; 20 世纪 90 年代初, 大连理工大学钟万协院士借鉴最 优控制领域的“状态空间法”, 通过引入对偶向量, 建 立了一套针对 Hamilton 动力学系统的辛几何方法理 论 $^{[10]}$; 近年来, 邓子辰教授等人将辛方法引入到非 线性动力系统中, 更进一步的拓展了 “保辛”思想的 应用领域 ${ }^{[13,14]}$. 在天体动力学与航天动力学领域, 辛 算法由于其优异的“保辛”特性得到了广泛的应用: 用辛算法研究天体运动中的误差累计问题, 可以控 制天体位置误差的快速增长, 并保持轨道稳定性 ${ }^{[15]}$; 将辛算法应用在近地小行星轨道演化数值研究中, 提高了计算精度, 同时很好地保持相流的各种重要 特征 ${ }^{[16]}$; 用辛算法计算卫星交会对接的动力学问题, 保持了飞行器轨迹的固有特性 ${ }^{[17]}$.

鉴于 Hamilton 系统辛算法保持系统定性特性的 优势, 本文根据已有研究成果, 在 Hamilton 体系下建 立太阳帆塔系统动力学方程, 利用辛 Runge-Kutta 方 法求解, 通过与传统的四阶 Runge-Kutta 方法对比, 研究了辛方法在长时间数值积分问题中的保辛特性, 并且利用该方法研究了太阳帆塔系统的平衡点及其 稳定性.

\section{2 太阳帆塔系统动力学模型}

太阳帆塔空间太阳能电站概念(图 1)的设计基础 是欧美提出的太阳帆技术和太阳塔概念. 该系统采 用重力梯度稳定方式, 使中央缆绳自动保持垂直于 地面, 以保证末端的发射天线对准地面 ${ }^{[2]}$. 太阳帆塔 在近地轨道进行组装, 然后通过电推力器将其送到 地球同步轨道. 在其运行过程中, 为了保证发电效率, 帆面始终保持与轨道平面垂直. 为简单起见, 本文只 研究太阳帆塔的一个单元, 即由两块太阳帆板和中 间系绳组成, 且只研究系统在轨道平面内的运动, 忽 略太阳帆板的变形. 该简化模型可以反映系统的结 构特征, 同时便于理论推导和定性研究.

如图 2, 将太阳帆塔中电池阵模块抽象成具有转 动惯量的刚杆, 这些刚杆由无质量的系绳连接, 将系 绳简单地视为弹簧. 为了研究太阳帆塔系统的动力 学问题, 假定系统在平面轨道上做面内运动, 只受到 地球中心引力场的作用, 忽略大气阻力, 太阳辐射压 力等摄动力的影响, 建立固定于地心 $O$ 的惯性坐标系 $O X Y$, 其中 $O X$ 轴位于赤道面内并指向春分点. 本文考 虑如图 2 所示的太阳帆塔简化结构模型, 刚杆长均为 $l$, 系绳是原长为 $L_{k}$ 弹性系数为 $k$ 的弹性绳, 刚杆线密 度为 $\rho$, 系统有 6 个自由度, 系统的广义坐标选为 $\boldsymbol{q}=\left[q_{1}, q_{2}, q_{3}, q_{4}, q_{5}, q_{6}\right]=\left[r_{1}, \theta_{1}, \alpha_{1}, r_{2}, \theta_{2}, \alpha_{2}\right]^{\mathrm{T}}$.

根据以上假设, 用 $\left(x_{1}, y_{1}\right),\left(x_{2}, y_{2}\right)$ 分别表示杆 $\mathrm{AB}$, $\mathrm{CD}$ 在坐标系 $O X Y$ 中的质心坐标, 则有:

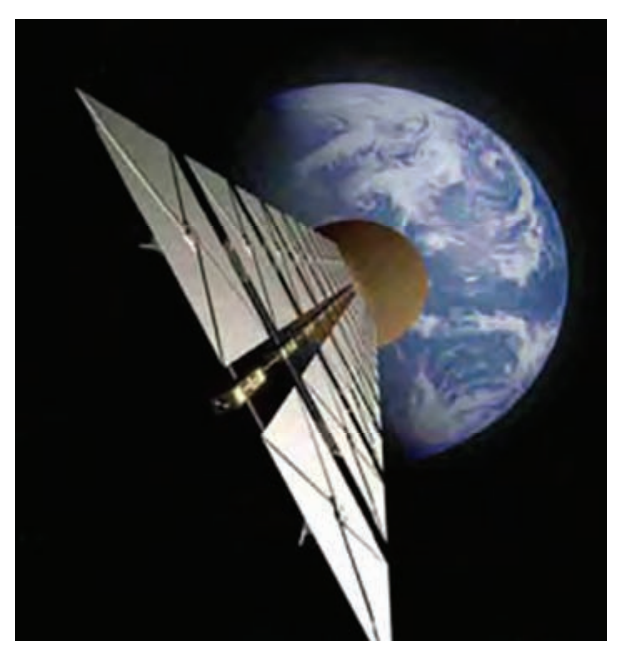

图 1 (网络版彩图)太阳帆塔太阳能电站概念 ${ }^{[18]}$ 


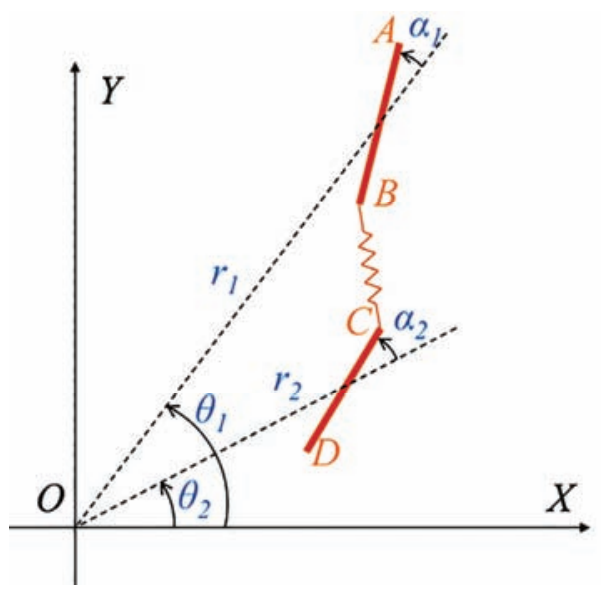

图 2 (网络版彩图)太阳帆塔简化结构模型

$$
\begin{gathered}
\left\{\begin{array}{l}
\left(x_{1}, y_{1}\right)=\left(r_{1} \cos \theta_{1}, r_{1} \sin \theta_{1}\right), \\
\left(x_{2}, y_{2}\right)=\left(r_{2} \cos \theta_{2}, r_{2} \sin \theta_{2}\right),
\end{array}\right. \\
\left\{\begin{array}{l}
\dot{x}_{1}=\dot{r}_{1} \cos \theta_{1}-r_{1} \dot{\theta}_{1} \sin \theta_{1}, \\
\dot{y}_{1}=\dot{r}_{1} \sin \theta_{1}+r_{1} \dot{\theta}_{1} \cos \theta_{1}, \\
\dot{x}_{2}=\dot{r}_{2} \cos \theta_{2}-r_{2} \dot{\theta}_{2} \sin \theta_{2}, \\
\dot{y}_{2}=\dot{r}_{2} \sin \theta_{2}+r_{2} \dot{\theta}_{2} \cos \theta_{2} .
\end{array}\right.
\end{gathered}
$$

$$
\begin{aligned}
U_{k} & =\frac{1}{2} k\left\{\left[\left(x_{B}-x_{C}\right)^{2}+\left(y_{B}-y_{C}\right)^{2}\right]^{1 / 2}-L_{k}\right\}^{2} \\
& =\frac{1}{2} k\left\{\left[-r_{1} l \cos \left(\alpha_{1}\right)-2 r_{1} r_{2} \cos \left(\theta_{1}-\theta_{2}\right)-r_{1} l \cos \left(-\theta_{1}+\theta_{2}+\alpha_{2}\right)+r_{2} l \cos \left(\theta_{1}+\alpha_{1}-\theta_{2}\right)\right.\right. \\
& \left.\left.+\frac{l^{2}}{2} \cos \left(\theta_{1}+\alpha_{1}-\theta_{2}-\alpha_{2}\right)+r_{2} l \cos \alpha_{2}+r_{1}^{2}+\frac{l^{2}}{2}+r_{2}^{2}\right]^{1 / 2}-L_{k}\right\}^{2} .
\end{aligned}
$$

系统的万有引力势能如下:

$$
\begin{aligned}
U_{g}= & -\frac{\mu \rho l}{r_{1}}+\frac{\mu \rho l^{3}}{24 r_{1}^{3}}\left(1-3 \cos ^{2} \alpha_{1}\right) \\
& -\frac{\mu \rho l}{r_{2}}+\frac{\mu \rho l^{3}}{24 r_{2}^{3}}\left(1-3 \cos ^{2} \alpha_{2}\right),
\end{aligned}
$$

为了运用 Hamilton 系统, 引入广义动量 $\boldsymbol{p}=$ $\left[\begin{array}{llllll}p_{1} & p_{2} & p_{3} & p_{4} & p_{5} & p_{6}\end{array}\right]^{\mathrm{T}}$, 建立系统的运动微分 方程组. 根据 $L=T-U$ 得到系统的 Lagrange 函数,

式中, $\mu$ 为地球引力常数, 故系统势能为

$$
U=U_{k}+U_{g} .
$$

系统的转动惯量为 $I_{1}=I_{2}=\frac{\rho l^{3}}{12}$, 给出系统的平 动动能和转动动能分别为

$$
\begin{aligned}
& T_{\text {tran }}=\frac{1}{2} \rho l\left(\dot{x}_{1}^{2}+\dot{y}_{1}^{2}+\dot{x}_{2}{ }^{2}+\dot{y}_{2}{ }^{2}\right), \\
& T_{\text {rot }}=\frac{1}{2} I_{1}\left(\dot{\theta}_{1}+\dot{\alpha}_{1}\right)^{2}+\frac{1}{2} I_{2}\left(\dot{\theta}_{2}+\dot{\alpha}_{2}\right)^{2} .
\end{aligned}
$$

将式(2)代入式(3)得到太阳帆塔系统的总动能为

$$
\begin{aligned}
T= & T_{\text {tran }}+T_{\text {rot }} \\
= & \frac{1}{24} \rho l\left[\dot{\alpha}_{1} l^{2}+2 \dot{\alpha}_{1} l^{2} \dot{\theta}_{1}+\dot{\alpha}_{2} l^{2}+2 \dot{\alpha}_{2} l^{2} \dot{\theta}_{2}+\dot{\theta}_{1} l^{2}+\dot{\theta}_{2} l^{2}\right. \\
& \left.+12 r_{1}^{2} \dot{\theta}_{1}^{2}+12 r_{2}^{2} \dot{\theta}_{2}^{2}+12 \dot{r}_{1}^{2}+12 \dot{r}_{2}^{2}\right] .
\end{aligned}
$$

图 2 中 $B$ 点和 $C$ 点的坐标为

$$
\left\{\begin{array}{l}
x_{\mathrm{B}}=r_{1} \cos \theta_{1}-\frac{l}{2} \cos \left(\theta_{1}+\alpha_{1}\right), \\
y_{\mathrm{B}}=r_{1} \sin \theta_{1}-\frac{l}{2} \sin \left(\theta_{1}+\alpha_{1}\right), \\
x_{\mathrm{C}}=r_{2} \cos \theta_{2}+\frac{l}{2} \cos \left(\theta_{2}+\alpha_{2}\right), \\
y_{\mathrm{C}}=r_{2} \sin \theta_{2}+\frac{l}{2} \sin \left(\theta_{2}+\alpha_{2}\right),
\end{array}\right.
$$

则系统的弹性势能为
由 $p_{i}=\frac{\partial L}{\partial \dot{q}_{i}}$ 求得广义动量的表达式为

$$
\left\{\begin{array}{l}
p_{1}=\rho l \dot{r}_{1} \\
p_{2}=\frac{1}{12} \rho l\left(\dot{\alpha}_{1} l^{2}+\dot{\theta}_{1} l^{2}+12 r_{1}^{2} \dot{\theta}_{1}\right) \\
p_{3}=\frac{1}{12} \rho l^{3}\left(\dot{\theta}_{1}+\dot{\alpha}_{1}\right) \\
p_{4}=\rho l \dot{r}_{2} \\
p_{5}=\frac{1}{12} \rho l\left(\dot{\alpha}_{2} l^{2}+\dot{\theta}_{2} l^{2}+12 r_{2}^{2} \dot{\theta}_{2}\right) \\
p_{6}=\frac{1}{12} \rho l^{3}\left(\dot{\theta}_{2}+\dot{\alpha}_{2}\right)
\end{array}\right.
$$

由式(9)反求出广义速度的表达式为 


$$
\left\{\begin{array}{l}
\dot{q}_{1}=\frac{p_{1}}{\rho l}, \\
\dot{q}_{2}=\frac{p_{2}-p_{3}}{\rho l p_{1}^{2},} \\
\dot{q}_{3}=-\frac{l^{2} p_{2}-l^{2} p_{3}-12 p_{3} p_{1}^{2}}{\rho l^{3} p_{1}{ }^{2}}, \\
\dot{q}_{4}=\frac{p_{4}}{\rho l}, \\
\dot{q}_{5}=\frac{p_{5}-p_{6}}{\rho l p_{2}{ }^{2}}, \\
\dot{q}_{6}=-\frac{l^{2} p_{5}-l^{2} p_{6}-12 p_{6} p_{2}{ }^{2}}{\rho l^{3} p_{2}{ }^{2}} .
\end{array} .\right.
$$

由于保守系统的 Hamilton 函数 $H$ 不显含 $t$, 故 $\mathrm{d} H / \mathrm{d} t=\partial H / \partial t=0$ ，则对于这种定常约束系统 Hamilton 函数可以表示为 ${ }^{[19]}$

$$
H=T+U \text {. }
$$

将式(4), (6), (7), (10)代入式(11)中, 可得到 Hamilton 函数的完整表达式:

$$
H=\frac{1}{24} \rho l\left[\dot{\alpha}_{1} l^{2}+2 \dot{\alpha}_{1} l^{2} \dot{\theta}_{1}+\dot{\alpha}_{2} l^{2}+2 \dot{\alpha}_{2} l^{2} \dot{\theta}_{2}+\dot{\theta}_{1} l^{2}\right.
$$

$$
\begin{aligned}
& \left.+\dot{\theta}_{2} l^{2}+12 r_{1}^{2} \dot{\theta}_{1}^{2}+12 r_{2}^{2} \dot{\theta}_{2}^{2}+12 \dot{r}_{1}^{2}+12 \dot{r}_{2}^{2}\right] \\
& +\frac{1}{2} k\left\{\left[-r_{1} l \cos \left(\alpha_{1}\right)-2 r_{1} r_{2} \cos \left(\theta_{1}-\theta_{2}\right)\right.\right. \\
& -r_{1} l \cos \left(-\theta_{1}+\theta_{2}+\alpha_{2}\right)+r_{2} l \cos \left(\theta_{1}+\alpha_{1}-\theta_{2}\right) \\
& +\frac{l^{2}}{2} \cos \left(\theta_{1}+\alpha_{1}-\theta_{2}-\alpha_{2}\right)+r_{2} l \cos \alpha_{2} \\
& \left.\left.+r_{1}^{2}+\frac{l^{2}}{2}+r_{2}^{2}\right]^{1 / 2}-L_{k}\right\} \\
& -\frac{\mu \rho l}{r_{1}}+\frac{\mu \rho l^{3}}{24 r_{1}^{3}}\left(1-3 \cos ^{2} \alpha_{1}\right) \\
& -\frac{\mu \rho l}{r_{2}}+\frac{\mu \rho l^{3}}{24 r_{2}^{3}}\left(1-3 \cos ^{2} \alpha_{2}\right) .
\end{aligned}
$$

给出 Hamilton 方程的标准形式:

$$
\left\{\begin{array}{l}
\dot{q}_{i}=\frac{\partial H}{\partial p_{i}}, \\
\dot{p}_{i}=-\frac{\partial H}{\partial q_{i}} .
\end{array}\right.
$$

由式(13)可以得到太阳帆塔系统的 Hamilton 方 程, 前 6 个方程是式(10), 后 6 个方程为

$$
\begin{aligned}
\dot{p}_{1} & =\frac{\left(p_{2}-p_{3}\right)^{2}}{l \rho r_{1}^{3}}-\frac{\mu \rho l}{r_{1}^{2}}+\frac{1}{8} \frac{\mu \rho l^{3}\left(1-3 \cos ^{2} \alpha_{1}\right)}{r_{1}^{4}} \\
& -\frac{1}{2} \frac{k\left(\sqrt{m}-L_{k}\right)\left(-l \cos \alpha_{1}-2 r_{2} \cos \left(\theta_{1}-\theta_{2}\right)-l \cos \left(-\theta_{1}+\theta_{2}+\alpha_{2}\right)+2 r_{1}\right)}{\sqrt{m}}, \\
\dot{p}_{2} & =-\frac{1}{2 \sqrt{m}}\left[k ( \sqrt { m } - L _ { k } ) \left(2 r_{1} r_{2} \sin \left(\theta_{1}-\theta_{2}\right)-r_{1} l \sin \left(-\theta_{1}+\theta_{2}+\alpha_{2}\right)\right.\right. \\
& \left.\left.-r_{2} l \sin \left(\theta_{1}+\alpha_{1}-\theta_{2}\right)-\frac{l^{2}}{2} \sin \left(\theta_{1}+\alpha_{1}-\theta_{2}-\alpha_{2}\right)\right)\right], \\
\dot{p}_{3}= & -\frac{1}{4} \frac{\mu \rho l^{3} \cos \alpha_{1} \sin \alpha_{1}}{r_{1}^{3}} \\
& -\frac{1}{2} \frac{k\left(\sqrt{m}-L_{k}\right)\left(r_{1} l \sin \alpha_{1}-r_{2} l \sin \left(\theta_{1}+\alpha_{1}-\theta_{2}\right)-\frac{1}{2} l^{2} \sin \left(\theta_{1}+\alpha_{1}-\theta_{2}-\alpha_{2}\right)\right)}{\sqrt{m}}, \\
\dot{p}_{4} & =\frac{\left(p_{5}-p_{6}\right)^{2}}{l \rho r_{2}^{3}}-\frac{\mu \rho l}{r_{2}^{2}}+\frac{1}{8} \frac{\mu \rho l^{3}\left(1-3 \cos ^{2} \alpha_{2}\right)}{r_{2}^{4}} \\
& -\frac{1}{2} \frac{k\left(\sqrt{m}-L_{k}\right)\left(l \cos \alpha_{2}-2 r_{1} \cos \left(\theta_{1}-\theta_{2}\right)+l \cos \left(\theta_{1}+\alpha_{1}-\theta_{2}\right)+2 r_{2}\right)}{\sqrt{m}}, \\
\dot{p}_{5}= & -\frac{1}{2 \sqrt{m}}\left[k ( \sqrt { m } - L _ { k } ) \left(-2 r_{1} r_{2} \sin \left(\theta_{1}-\theta_{2}\right)+r_{1} l \sin \left(-\theta_{1}+\theta_{2}+\alpha_{2}\right)\right.\right. \\
& \left.\left.+r_{2} l \sin \left(\theta_{1}+\alpha_{1}-\theta_{2}\right)+\frac{l^{2}}{2} \sin \left(\theta_{1}+\alpha_{1}-\theta_{2}-\alpha_{2}\right)\right)\right],
\end{aligned}
$$




$$
\begin{aligned}
\dot{p}_{6} & =-\frac{1}{4} \frac{\mu \rho l^{3} \cos \alpha_{2} \sin \alpha_{2}}{r_{2}^{3}} \\
& -\frac{1}{2} \frac{k\left(\sqrt{m}-L_{k}\right)\left(-r_{2} l \sin \alpha_{2}+r_{1} l \sin \left(\theta_{2}+\alpha_{2}-\theta_{1}\right)+\frac{1}{2} l^{2} \sin \left(\theta_{1}+\alpha_{1}-\theta_{2}-\alpha_{2}\right)\right)}{\sqrt{m}},
\end{aligned}
$$

其中,

$$
\begin{aligned}
m= & -r_{1} l \cos \alpha_{1}-2 r_{1} r_{2} \cos \left(\theta_{1}-\theta_{2}\right)-r_{1} l \cos \left(-\theta_{1}+\theta_{2}+\alpha_{2}\right)+r_{2} l \cos \left(\theta_{1}+\alpha_{1}-\theta_{2}\right) \\
& +\frac{1}{2} l^{2} \cos \left(\theta_{1}+\alpha_{1}-\theta_{2}-\alpha_{2}\right)+r_{2} l \cos \alpha_{2}+r_{1}^{2}+\frac{1}{2} l^{2}+r_{2}^{2} .
\end{aligned}
$$

式(10)和(14)即为太阳帆塔简化模型的 Hamilton 方程.

\section{3 辛 Runge-Kutta 方法求解太阳帆塔系统 方程}

\section{1 辛 Runge-Kutta 格式}

$s$ 级的隐式 Runge-Kutta 方法的一般格式为

$$
\left\{\begin{array}{l}
\boldsymbol{u}_{n+1}=\boldsymbol{u}_{n}+\tau \sum_{i=1}^{s} c_{i} \boldsymbol{k}_{i}, \\
\boldsymbol{k}_{i}=f\left(t_{n}+a_{i} \tau, \boldsymbol{u}_{n}+\tau \sum_{j=1}^{s} b_{i j} \boldsymbol{k}_{j}\right),(r=1,2, \cdots, s),
\end{array}\right.
$$

式中, $i, j=1,2, \cdots, s, c_{j} \geqslant 0, \sum_{i=1}^{s} c_{i}=1, \sum_{j=1}^{s} b_{i j}=a_{i}$, $\sum_{j=1}^{s} a_{j}=1, \tau$ 为计算步长, Sanz-Serna, Lasa-gni 和 Suris ${ }^{[20]}$ 从不同角度证明, 上述 Runge-Kutta 方法的系 数满足以下条件时为辛 Runge-Kutta 方法:

$$
c_{i} c_{j}-b_{i j} c_{i}-b_{j i} c_{j}=0, \quad(i, j=1 \cdots \cdots s) .
$$

辛 Runge-Kutta 方法是一种构造辛算法的有效途 径, 优点是构造简单, 适用范围广, 因此得到广泛的 应用. 系数 $b_{i j}$ 和 $c_{i}$ 取不同的值时, 可得到不同的辛 Runge-Kutta 方法. 常用的一种二级四阶辛 RungeKutta 格式为

$$
\begin{aligned}
& B=\left[\begin{array}{ll}
b_{11} & b_{12} \\
b_{21} & b_{22}
\end{array}\right]=\left[\begin{array}{cc}
\frac{1}{4} & \frac{1}{4}-\frac{\sqrt{3}}{6} \\
\frac{1}{4}+\frac{\sqrt{3}}{6} & \frac{1}{4}
\end{array}\right], \\
& {\left[\begin{array}{ll}
c_{1} & c_{2}
\end{array}\right]=\left[\begin{array}{ll}
\frac{1}{2} & \frac{1}{2}
\end{array}\right] .}
\end{aligned}
$$

\section{2 动力学仿真分析}

在 Hamilton 系统下使用辛算法能保持系统的很 多定性特性, 如它的相流形能保持相空间的面积和 体积不变, 以及动量和能量不变 ${ }^{[9]}$. 而采用传统的非 辛算法, 则不能保持 Hamilton 系统的定性特性不变, 随着积分时间的增加, 误差累积, 系统的能量、动量 等定性性质都会随着时间发生改变 ${ }^{[17]}$. 基于模型假 设, 太阳帆塔系统在空间运行时, 能量是守恒的.

下面将采用辛 Runge-Kutta 方法和传统的四阶 Runge-Kutta 方法求解上述 Hamilton 方程, 并作结果分 析. 根据欧洲太阳帆塔空间太阳能电站的设计数据 ${ }^{[4]}$, 地球引力常数 $\mu=3.98603 \times 10^{14}, L_{k}=100 \mathrm{~m}, \rho=21.333$ $\mathrm{kg} / \mathrm{m}, l=150 \mathrm{~m}$, 令系统在近圆轨道运行, 轨道半径 $r_{2}=6574.330 \mathrm{~km}, \quad r_{1}=r_{2}+L_{k}+l, \dot{\theta}=\sqrt{\mu\left(\frac{r_{1}+r_{2}}{2}\right)^{-3}}$, 则初值取为

$$
\begin{aligned}
\boldsymbol{x}_{\mathbf{0}}= & {\left[\boldsymbol{q}_{\mathbf{0}} ; \boldsymbol{p}_{\mathbf{0}}\right], } \\
\boldsymbol{q}_{0}= & {\left[\mathrm{r}_{1}, 0,0, r_{2}, 0,0\right]^{\mathrm{T}}, \dot{\boldsymbol{q}}_{0}=[0, \dot{\theta}, 0,0, \dot{\theta}, 0]^{\mathrm{T}}, } \\
\boldsymbol{p}_{\mathbf{0}}= & {[0,163819898572397,7106.10951378213,} \\
& 0,163807440226471,7106.10951378213]^{\mathrm{T}} .
\end{aligned}
$$

图 3(a)和(b)表示用传统的 Runge-Kutta 方法计算 得到步长取不同值时的相对能量误差, 图 3(c)和(d) 表示用传统的 Runge-Kutta 方法计算得到绳长改变量 随时间的变化图. 通过对比可以看出, 取小步长时, 传统的 Runge-Kutta 方法计算的绳长变化量是在小范 围内稳定变化的, 相对能量误差随时间线性增长. 而 取大步长时, 传统的 Runge-Kutta 方法计算的绳子伸 长量逐渐减小, 到最后几乎停止了振荡, 相对能量误 差开始是线性增长的, 随着系统振荡的停止, 相对能 量误差开始稳定在一个值, 不再发生较大的变化. 

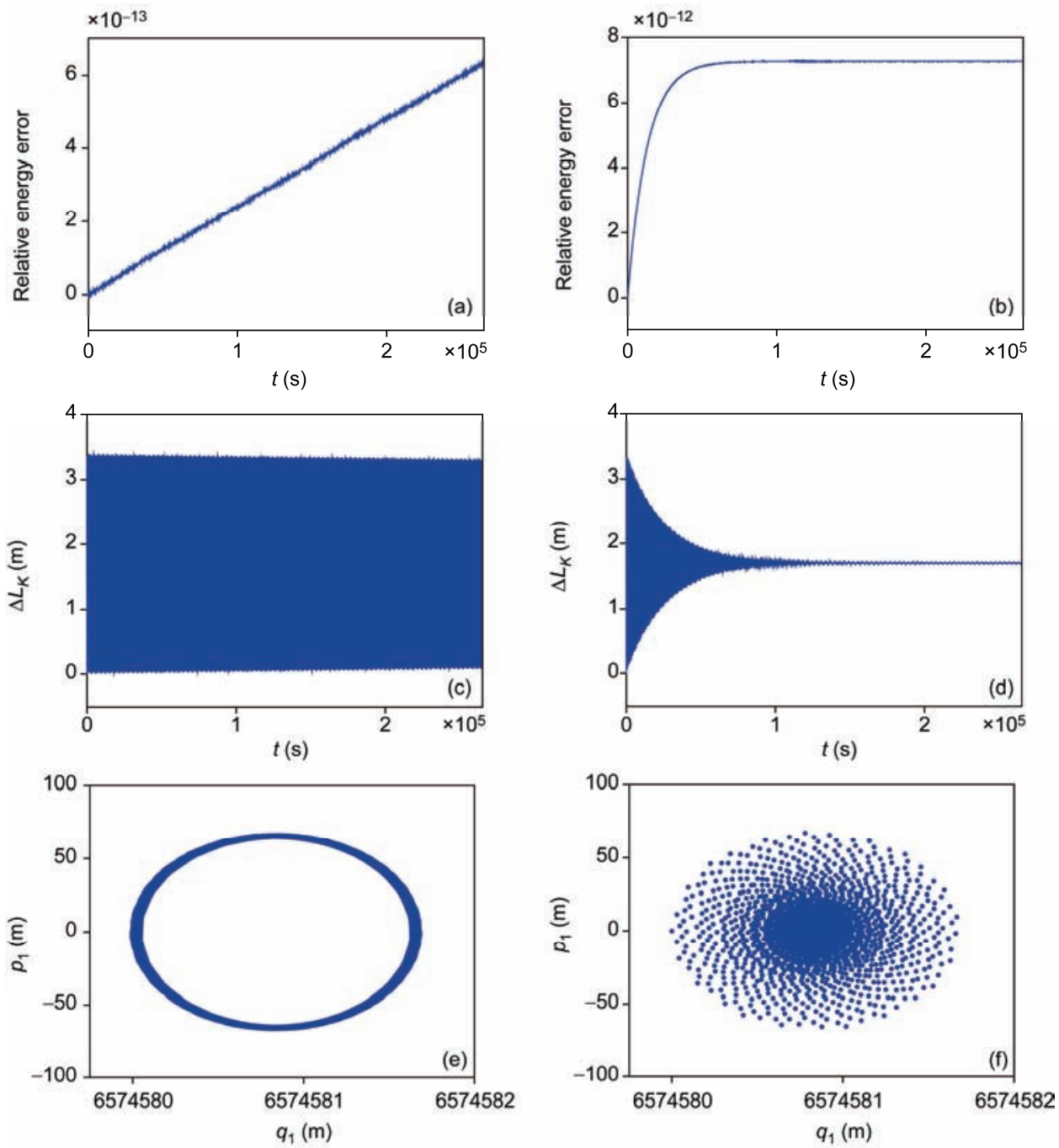

图 3 (网络版彩图)传统的 Runge-Kutta 方法, $k=1$

(a) 相对能量误差变化图, $\tau=10 \mathrm{~s}$; (b) 相对能量误差变化图, $\tau=30 \mathrm{~s}$; (c) 弹簧伸长量变化图, $\tau=10 \mathrm{~s}$; (d) 弹簧伸长量变化图, $\tau=30 \mathrm{~s}$; (e) 对偶空 间相图, $\tau=10 \mathrm{~s}$; (f) 对偶空间相图, $\tau=30 \mathrm{~s}$

图 3(e)和(f)表示用传统的 Runge-Kutta 方法计算 得到太阳帆塔系统相图. 对比图 3(e)和(f)可以看出, 小步长时, 传统的 Runge-Kutta 方法计算的相空间的 面积不变, 但步长增大后, 相空间的面积不再保持不 变.

通过对以上结果的分析, 可以发现: 传统的 Runge-Kutta 方法存在算法内在阻尼, 将弹簧的振荡 效果耗散掉了, 且无法保持系绳弹性振动的固有特 性和相空间的面积不变.

图 4(a)和(b)表示用辛 Runge-Kutta 方法计算所得 取不同步长时的相对能量误差. 对比图 4(a)和(b)可 以看出, 随着步长增大, 辛 Runge-Kutta 方法计算的
相对能量误差仍然随时间作周期性变化, 且其幅值 没有明显改变, 稳定性很好.

图 4(c)和(d)表示, 用辛 Runge-Kutta 方法计算得 到绳长改变量随时间的变化图. 对比图 4(c)和(d)可 以看出, 辛 Runge-Kutta 方法计算的绳长变化量, 在 取不同步长时都呈周期性变化, 变化幅值较小, 很好 地保持了无阻尼系统弹性振动的固有特性, 有利于 实际空间动力学研究应用. 图 4(e)和(f)表示, 用辛 Runge-Kutta 方法计算得到太阳帆塔系统相图. 对比 图 4(e)和(f)可以看出, 辛 Runge-Kutta 方法能够保持 相空间的面积不变.

以上结果表明: 辛 Runge-Kutta 方法计算的相对 

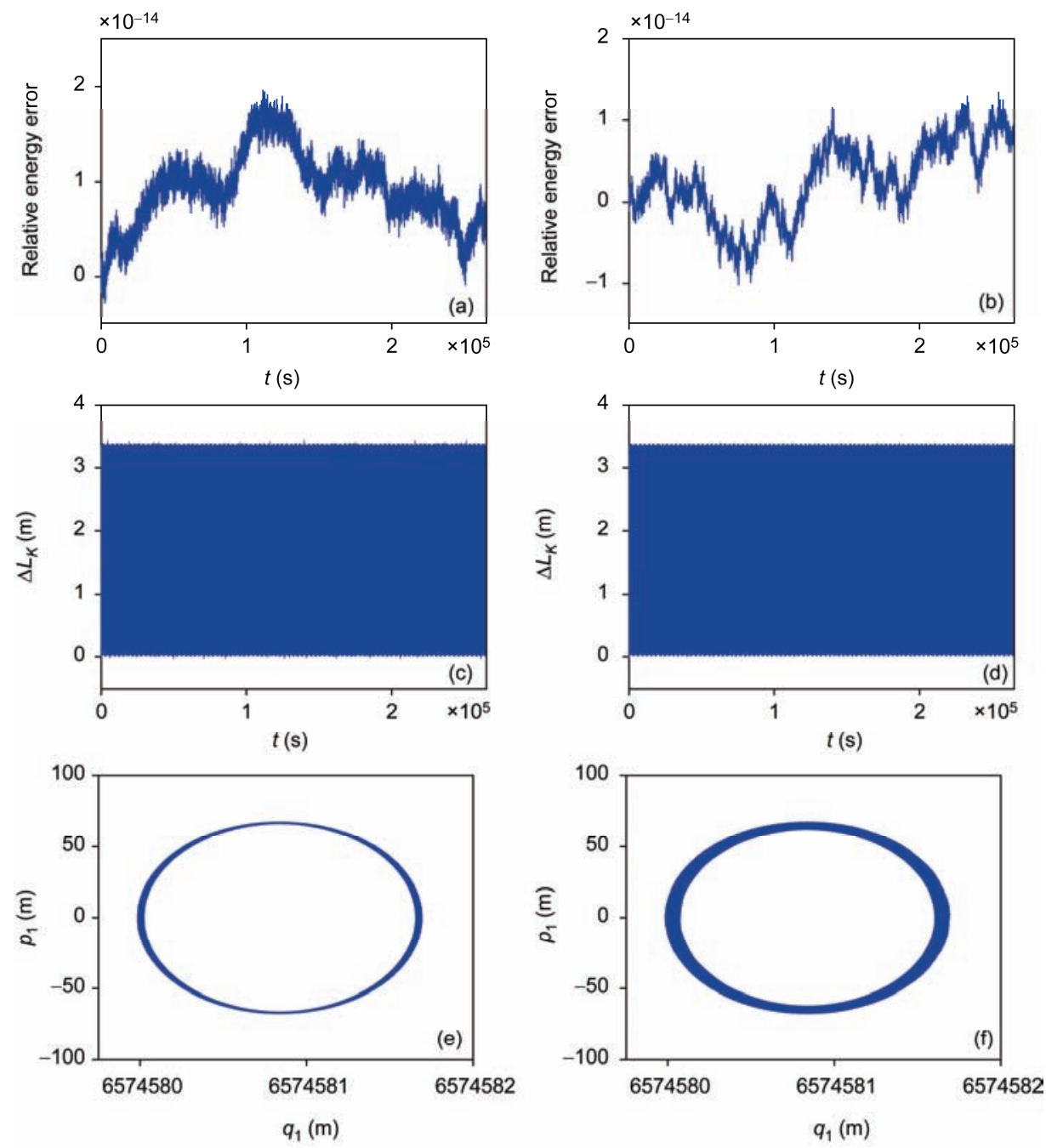

图 4 (网络版彩图)辛 Runge-Kutta 方法, $k=1$

(a) 相对能量误差变化图, $\tau=10 \mathrm{~s}$; (b) 相对能量误差变化图, $\tau=30 \mathrm{~s}$; (c) 弹簧伸长量变化图, $\tau=10 \mathrm{~s}$; (d) 弹簧伸长量变化图, $\tau=30 \mathrm{~s}$; (e) 对偶空 间相图, $\tau=10 \mathrm{~s}$; (f) 对偶空间相图, $\tau=30 \mathrm{~s}$

能量误差比传统的 Runge-Kutta 方法量级小 3 个数量 级, 稳定性明显增强, 保能量效果更好, 而且很好地 保持了无阻尼系统弹性振动的固有特性和相空间的 面积不变. 可见辛 Runge-Kutta 方法在实际工程中有 更好的应用价值，体现了该方面的优越性.

\section{4 平衡点与稳定性}

\section{1 求解太阳帆塔系统的平衡点}

为了使太阳帆塔系统能持续地搜集太阳能、向地 面定点传送微波能量, 系统需要稳定地保持垂直于
地面的姿态, 因此研究太阳帆塔系统在平衡位置的 运行状况及稳定性, 对于设计目的有着重要的价值. 通过力学分析, 推测太阳帆塔动力学系统可能存在 沿轨道径向的平衡位置. 稳定性是判断系统运动特 性的重要标准. 实际工程应用中的每一个系统都必 须是稳定的, 不稳定的系统付诸工程实践是具有破 坏性的、不可实施的 ${ }^{[21]}$, 而关于力学系统的稳定应用 在实际工程中更是必不可少的. 因此研究太阳帆塔 系统平衡位置的稳定性具有重要的理论意义和实际 应用价值.

对整个太阳帆塔系统而言, 所受的外力有: 绳子 


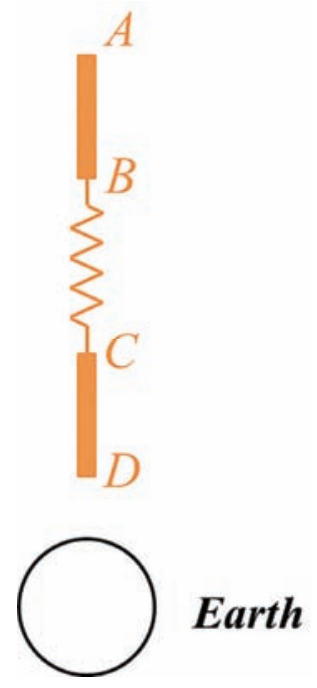

图 5 (网络版彩图)太阳帆塔简化结构模型的平衡点位置

拉力 $T$, 万有引力 $G=\frac{\mu m}{r^{2}}$, 要使系统达到平衡态, 需 满足 $\dot{\theta}_{1}=\dot{\theta}_{2}=\dot{\theta}, \alpha_{1}=\alpha_{2}=0, \theta_{1}=\theta_{2}$. 故在系统平衡 态时有:

$$
\left\{\begin{array}{l}
G_{1}+T=F_{1}, \\
G_{2}-T=F_{2}, \\
k\left(r_{1}-r_{2}-l-L_{k}\right)=T,
\end{array}\right.
$$

式中, $G_{i}, F_{i}(i=1,2)$ 分别表示万有引力和离心力, 其 表达式如下:

$$
\left\{\begin{array}{l}
G_{i}=\frac{\mu m}{r^{2}}=\int_{0}^{l} \frac{\mu \rho}{\left(r-\frac{l}{2}+x\right)^{2}} \mathrm{~d} x=\frac{\mu \rho l}{r^{2}-\frac{l^{2}}{4}}, \\
F_{i}=m r \dot{\theta}^{2}=\int_{0}^{l} \rho \dot{\theta}^{2}\left(r-\frac{l}{2}+x\right) \mathrm{d} x=\rho \dot{\theta}^{2} r l .
\end{array}\right.
$$

通过式(19)和(20)解得:

$$
\left\{\begin{array}{l}
\dot{\theta}=\sqrt{\frac{\mu}{r_{1}+r_{2}}\left(\frac{1}{r_{1}^{2}-\frac{l^{2}}{4}}+\frac{1}{r_{2}^{2}-\frac{l^{2}}{4}}\right)} \\
T=\rho \dot{\theta}^{2} r_{1} l-\mu \rho \frac{l}{r_{1}^{2}-\frac{l^{2}}{4}}, \\
L_{k}=r_{1}-r_{2}-l-\frac{T}{k} .
\end{array}\right.
$$

其他参数取值如下: $r_{2}=6574.330 \mathrm{~km}, r_{1}=r_{2}+250$, $l=150 \mathrm{~m}, k=1$, 则得到平衡点:

$$
\begin{aligned}
& \boldsymbol{x}_{\mathrm{s}}=\left[\boldsymbol{q}_{\mathrm{s}} ; \boldsymbol{p}_{\mathrm{s}}\right], \\
& \boldsymbol{q}_{\mathrm{s}}=\left[r_{1}, \dot{\theta} t, 0, r_{2}, \dot{\theta} t, 0\right]^{\mathrm{T}}, \dot{\boldsymbol{q}_{\mathrm{s}}}=[0, \dot{\theta}, 0,0, \dot{\theta}, 0]^{\mathrm{T}}, \\
& \boldsymbol{p}_{\mathrm{s}}=[0,163819898671886,7106.10951809773, \\
& 0,163807440325953,7106.10951809773]^{\mathrm{T}} .
\end{aligned}
$$

为了验证平衡点的正确性, 将式(22)平衡点的数 值代入 Hamilton 方程 (10) 和 (14) 中, 得到 $\dot{\boldsymbol{p}}, \dot{q}_{1}, \dot{q}_{3}, \dot{q}_{4}, \dot{q}_{6}$ 都为零, $\dot{q}_{2}, \dot{q}_{5}$ 为常值, 说明所求 平衡点的值是正确的.

\section{2 辛 Runge-Kutta 方法验证平衡位置的必要性}

由于论文 2.2 小节已经证明了辛 Runge-Kutta 方 法在保持系统固有特性方面的优势, 下面将分别取 式(18)所给初值和式(22)所求平衡点初值, 对太阳帆 塔简化模型的位置, 姿态运动仿真, 得到图 6 .

图 6 表示两种初值计算得到两刚性杆轨道差 $r_{1}-r_{2}$ 的相图, 真近点角差 $\theta_{1}-\theta_{2}$ 的相图, 姿态角 $\alpha_{1}$ 和 $\alpha_{2}$ 的相图. 从图中可以看出: 取非平衡点计算的轨道 差、真近点角差值、姿态角变化范围都较大, 且很不 规律; 而取平衡点计算的轨道差、真近点角差值、姿 态角都几乎稳定在一个定值.

以上结果表明: 考虑绳子弹性的情况下, 太阳帆 塔在万有引力梯度的作用下, 如果系统处于非平衡 点, 轨道半径、真近点角和姿态角都会出现振荡. 姿 态角的振荡会影响太阳帆塔缆绳底部的发射天线的 对地指向精度, 从而影响系统的正常工作. 因此为了 使系统能精确地实现对地指向功能, 有必要将系统 放在平衡点附近运动, 并对平衡点的稳定性作深入 的研究.

\section{3 理论分析平衡点的稳定性并对其进行数值 验证}

为了研究太阳帆塔系统在平衡点附近的稳定性, 考虑系统的 Hamilton 方程, 其状态空间表达式为

$$
\dot{x}=f(\mathrm{x}) .
$$

将式(23)在平衡点 $\boldsymbol{x}_{\mathrm{s}}$ 处线性化得到:

$$
\dot{\tilde{x}}=\boldsymbol{D f}(\boldsymbol{x}) \cdot \tilde{\boldsymbol{x}},
$$

式中, $\tilde{x}=x-x_{s}, D f(x)$ 是向量函数 $\boldsymbol{f}(\boldsymbol{x})$ 在平衡 

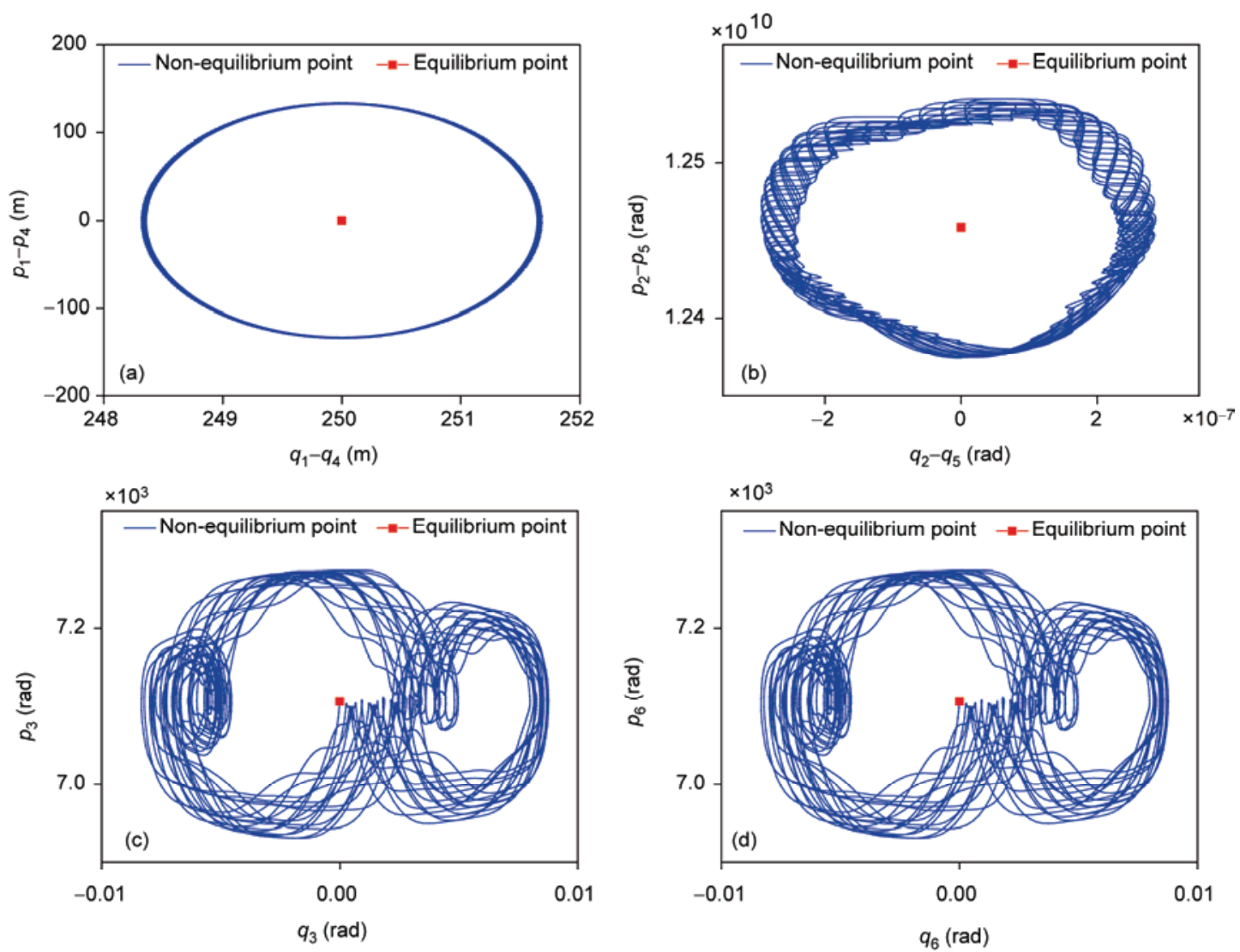

图 6 (网络版彩图)非平衡点和平衡点, $\tau=10 \mathrm{~s}$

(a) 轨道差值相图; (b) 真近点角差值相图; (c) 姿态角 $\alpha_{1}$ 相图; (d) 姿态角 $\alpha_{2}$ 相图

点 $\boldsymbol{x}_{s}=\left[\boldsymbol{q}_{s} ; \boldsymbol{p}_{s}\right]$ 附近的 Jacobi 矩阵, 由于文章篇幅原 因, $\boldsymbol{D f}(\boldsymbol{x})$ 具体数值见附录.

通过求解常值矩阵 $D f(x)$ 的特征值, 判断平衡点 $x_{s}$ 的稳定性.

下面给出判断线性化方程的 Lyapunov 稳定性判 定定理 ${ }^{[22]}$ :

(1) 零解是渐进稳定的充要条件是矩阵 $D f(x)$ 的 全部特征值具有负的实部;

(2) 零解是稳定的充要条件是矩阵 $D f(x)$ 的全部 特征值具有非正的实部, 并且所有实部为零的特征 值所对应的若当块都是一阶的;

(3) 零解是不稳定的充要条件是矩阵 $D f(x)$ 的特 征值中至少有一个实部为正, 或者至少有一个实部 为零且所对应的若当块是高于一阶的.

当太阳帆塔系统在平衡点 $\boldsymbol{x}_{s}=\left[\boldsymbol{q}_{s} ; \boldsymbol{p}_{s}\right]$ 处取值时, 求得矩阵 $D f(x)$ 的特征值为

$$
\left\{\begin{array}{l}
\lambda_{1,2}= \pm 0.025030060242936 i \\
\lambda_{3,4}= \pm 0.007940598121848 i \\
\lambda_{5,6}= \pm 0.001184351482427 i \\
\lambda_{7,8}= \pm 0.002043065147694 i \\
\lambda_{9,10}= \pm 0.005024757809575 i \\
\lambda_{11,12}= \pm 0.000000000001345 i
\end{array}\right.
$$

所求式(25)的特征值所有实部为零, 且这些特征 值所对应的若当块也都是一阶的, 故沿轨道径向的 平衡位置是稳定的.

下面用辛 Runge-Kutta 方法对太阳帆塔系统平衡 位置的稳定性进行数值验证. 图 7 描绘了系统在 $O X Y$ 坐标系下, 在平衡位置给予小的摄动以后太阳帆塔 系统的运行情况, 此时 $\dot{\boldsymbol{q}}_{s}=[0,0.00118435158634962$, $0.001,0,0.00118435158634962,0]^{\mathrm{T}}$.

从图 7(a)可以看出, 给系统小的扰动后, 系绳随 时间作周期性变化, 变化幅值很小; 图 7(b)反映出轨 

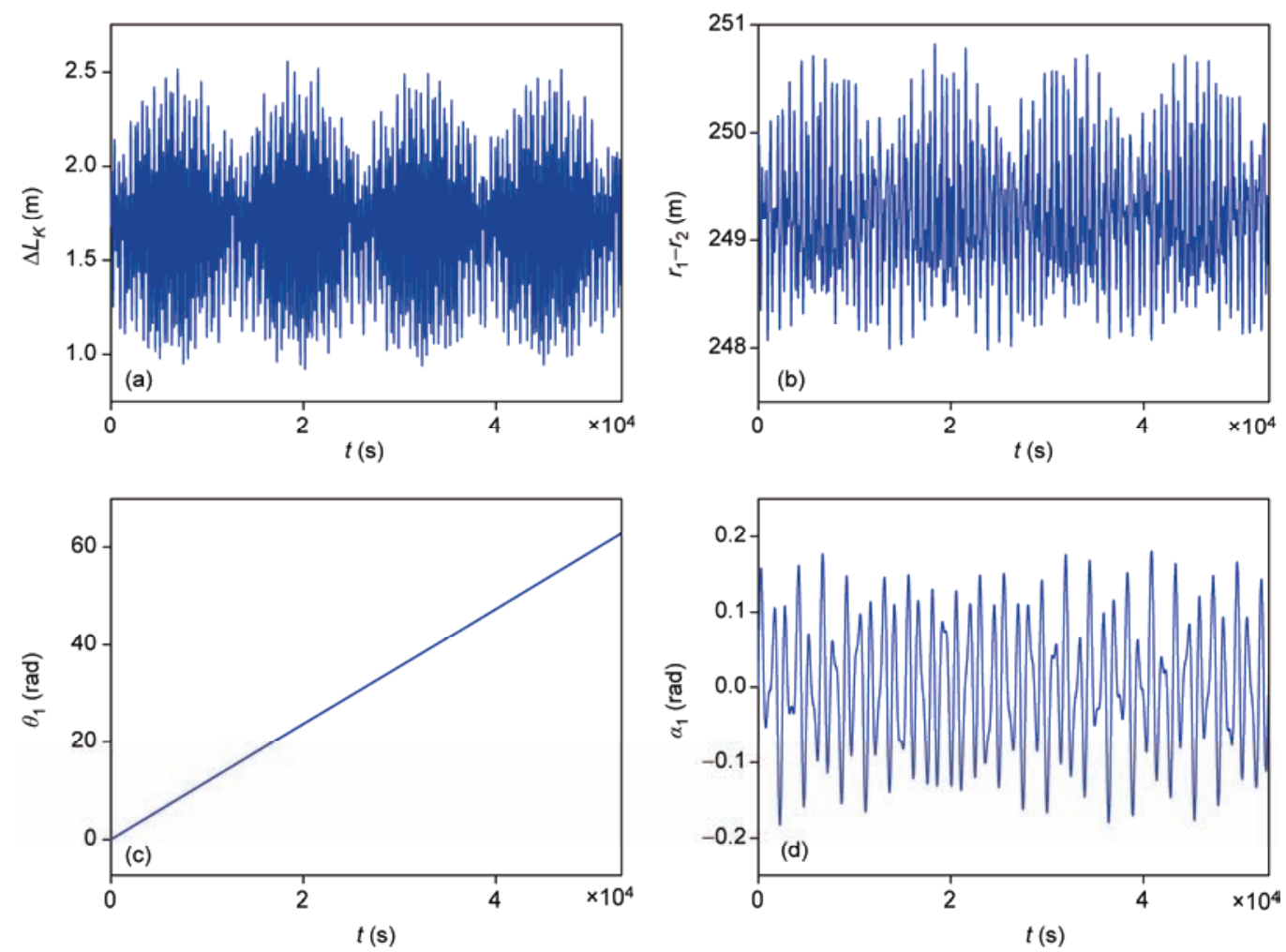

图 7 (网络版彩图)平衡位置 $x_{s}=\left[\boldsymbol{q}_{s} ; \boldsymbol{p}_{\mathrm{s}}\right], \tau=10 \mathrm{~s}$

道半径差值随时间发生小幅的稳定振荡, 振幅始终 小于 $3.3 \mathrm{~m}$; 图 7(c)和(d)分别表示一根刚杆轨道转角 和姿态角随时间的变化, 真近点角随着时间线性增 长, 且姿态角在小范围内做周期性振荡, 振幅始终小 于 $0.4 \mathrm{rad} / \mathrm{s}$. 可见, 本文系统既不发散也不收玫, 只 在平衡位置附近做小幅振荡, 证明太阳帆塔系统沿 轨道径向的平衡位置是稳定的.

本文根据 Lyapunov 稳定性判定定理，通过理论 推导和数值验证充分说明: 太阳帆塔系统存在沿轨 道径向的平衡位置, 且该平衡位置是稳定的.

\section{5 结论}

本文基于 Hamilton 体系建立了太阳帆塔简化模 型在轨运行的动力学方程, 并采用保辛算法和非辛 算法进行动力学仿真计算, 得到如下结论.

(1) 辛 Runge-Kutta 方法计算的相对能量误差量 级更小, 随时间周期性改变, 且在取不同步长时能够 保持系绳弹性振动的固有特性和相空间的面积不变, 而传统的四阶 Runge-Kutta 方法计算的相对能量误差
随时间线性增长，同时不能保持系统的固有性质

(2) 发现太阳帆塔在万有引力梯度的作用下，系 统处于非平衡点, 轨道半径、真近点角和姿态角都会 出现振荡, 从而影响太阳帆塔缆绳底部的发射天线 的对地指向精度, 以此证明研究平衡位置的必要性.

(3) 证明了太阳帆塔系统沿径向的平衡位置是 稳定的，这一结论对于利用太阳帆塔稳定收集太阳 能，向地面定点传输能源等具有很重要的应用价值.

\section{附录}

这里只给出矩阵 $\boldsymbol{D} \boldsymbol{f}$ 的非零元素, 其中 $\boldsymbol{D} \boldsymbol{f}_{i, j}$ 代表 矩阵 $\boldsymbol{D} \boldsymbol{f}$ 的第 $i$ 行、第 $j$ 列的元素，则矩阵 $\boldsymbol{D} \boldsymbol{f}$ 的非零 元素为

$$
\begin{aligned}
& -\boldsymbol{D} \boldsymbol{f}_{2,1}=\boldsymbol{D} \boldsymbol{f}_{3,1}=\boldsymbol{D} \boldsymbol{f}_{7,8}=\boldsymbol{D} \boldsymbol{f}_{7,9}=3.60295752 \times 10^{-10}, \\
& -\boldsymbol{D} \boldsymbol{f}_{5,4}=\boldsymbol{D} \boldsymbol{f}_{6,4}=\boldsymbol{D} \boldsymbol{f}_{10,11}=\boldsymbol{D} \boldsymbol{f}_{10,12}=3.60282052 \times 10^{-10}, \\
& \boldsymbol{D} \boldsymbol{f}_{7,1}=\boldsymbol{D} \boldsymbol{f}_{10,4}=-1.0044891150, \\
& \boldsymbol{D} \boldsymbol{f}_{7,4}=\boldsymbol{D} \boldsymbol{f}_{10,1}=1, \\
& -\boldsymbol{D} \boldsymbol{f}_{8,2}=\boldsymbol{D} \boldsymbol{f}_{8,5}=\boldsymbol{D} \boldsymbol{f}_{11,2}=-\boldsymbol{D} \boldsymbol{f}_{11,5}=7.275415765 \times 10^{11}, \\
& \boldsymbol{D} \boldsymbol{f}_{8,3}=\boldsymbol{D} \boldsymbol{f}_{9,2}=-\boldsymbol{D} \boldsymbol{f}_{9,5}=-\boldsymbol{D} \boldsymbol{f}_{11,3}=8.29957818 \times 10^{6},
\end{aligned}
$$




$$
\begin{aligned}
& D \boldsymbol{f}_{8,6}=\boldsymbol{D} \boldsymbol{f}_{12,2}=-\boldsymbol{D} \boldsymbol{f}_{11,6}=-D \boldsymbol{f}_{12,5}=8.29970442 \times 10^{6}, \quad \boldsymbol{D} \boldsymbol{f}_{1,7}=D \boldsymbol{f}_{4,10}=0.0003125 \text {, } \\
& \boldsymbol{D} \boldsymbol{f}_{9,3}=-246.1682686, \quad \quad \boldsymbol{D f _ { 2 , 8 }}=-\boldsymbol{D} \boldsymbol{f}_{2,9}=-\boldsymbol{D} \boldsymbol{f}_{3,8}=7.229595403 \times 10^{-18} \text {, } \\
& \boldsymbol{D} \boldsymbol{f}_{9,6}=D \boldsymbol{f}_{12,3}=-94.6805625, \quad \quad \boldsymbol{D \boldsymbol { f } _ { 3 , 9 }}=\boldsymbol{D} \boldsymbol{f}_{6,12}=1.6666666667 \times 10^{-7} \text {, } \\
& \boldsymbol{D} \boldsymbol{f}_{12,6}=-246.1711489 \text {, } \quad \boldsymbol{D} \boldsymbol{f}_{5,11}=-\boldsymbol{D} \boldsymbol{f}_{5,12}=-\boldsymbol{D} \boldsymbol{f}_{6,11}=7.23014525 \times 10^{-18} \text {. }
\end{aligned}
$$

\section{参考文献}

1 Glaser P E. Power from the sun: Its future. Science, 1968, 162: 857-861

2 侯欣宾. 不同空间太阳能电站概念方案的比较研究. 太阳能学报, 2012, S1: 63-69

3 侯欣宾, 王立，朱耀平，等. 国际空间太阳能电站发展现状. 太阳能学报, 2009, 10: 1263-1268

4 Seboldt W, Klimke M, Leipold M, et al. European Sail Tower SPS concept. Acta Astronaut, 2001, 48: 785-792

5 庄逢甘, 李明, 王立, 等. 未来航天与新能源的战略结合一一空间太阳能电站. 中国航天, 2008, 7: 36-39

6 夏洁. 两体绳系卫星系统的非线性振动与控制. 硕士学位论文. 南京: 南京航空航天大学, 2012.9-18

7 尤超蓝, 洪嘉振. 空间交会对接过程的动力学模型与仿真. 动力学与控制学报, 2004, 2: 25-30

8 赵将, 刘铖, 田强, 等. 黏弹性薄膜太阳帆自旋展开动力学分析. 力学学报, 2013, 5: 746-754

9 冯康, 秦孟兆. 哈密尔顿系统的辛几何算法. 杭州: 浙江科学技术出版社, 2003

10 钟万勋. 应用力学对偶体系. 北京: 科学出版社, 2002

11 Benner P, Kressner D, Mehrmann V. Structure preservation: A challenge in computational control. Future Gener Comp Sy, 2003, 19: 1243-1252

12 Feng K. On Difference Schemes and Symplectic Geometry. Beijing: Science Press, 1984. 42-58

13 Zhang S, Zhang M, Deng Z, et al. Solution of nonlinear dynamic differential equations based on numerical Laplace transform inversion. Appl Math Comput, 2007, 189: 79-86

14 张素英, 邓子辰. 非线性动力学系统的几何积分理论及应用. 西安: 西北工业大学出版社, 2005

15 刘林, 廖新浩, 赵长印, 等. 辛算法在动力天文中的应用(III). 天文学报, 1994, 1: 51-66

16 刘林, 廖新浩, 季江徽. 辛算法在近地小行星轨道演化数值研究中的应用. 计算物理, 1997, Z1: 268-270

17 李庆军, 叶学华, 王博, 等. 辛 Runge-Kutta 方法在卫星交会对接中的非线性动力学应用研究. 应用数学和力学, 2014, 12: 1299-1307

18 王立，侯欣宾. 空间太阳能电站的关键技术及发展建议. 航天器环境工程, 2014, 4: 343-350

19 应祖光. 高等动力学理论及应用. 杭州：浙江大学出版社, 2011

20 Lasagni F M. Canonical Runge-Kutta methods. Z Angew Math Phys, 1988, 39: 952-953

21 刘豹. 现代控制理论. 北京: 机械工业出版社, 1983

22 徐博侯, 曲绍兴. 非线性力学导论. 杭州: 浙江大学出版社, 2012 


\title{
Dynamic behavior of sail tower SPS based on the symplectic Runge-Kutta method
}

\author{
DENG ZiChen $^{1,2}$, CAO ShanShan ${ }^{1}$, LI QingJun ${ }^{1} \&$ JIANG XianHong ${ }^{1}$ \\ ${ }^{1}$ School of Mechanics, Civil Engineering and Architecture, Northwestern Polytechnical University, Xi' an 710072, China; \\ ${ }^{2}$ School of Natural and Applied Sciences, Northwestern Polytechnical University, Xi'an 710072, China
}

Space-based solar power as a promising approach to achieve clean and renewable energy had received extensive attention during the last several decades. The dynamics and stability of system of solar power satellite (SPS) in orbit were investigated through the application of Hamilton theory. First, the simplified structure model of sail tower SPS was built, and dynamic governing equation of simplified model of sail tower SPS in the Hamilton form were derived by introducing the concepts of generalized coordinates and generalized momentums and implementing variation method. Then, by numerically solving the equation through the application of the symplectic Runge-Kutta method and comparing the results with the traditional Runge-Kutta method, numerical examples demonstrated that the results achieved by the symplectic Runge-Kutta method indicated the energy preservation as well as the vibrational amplitude of the tether. Finally, the equilibrium points was calculated based on mechanical balance principle. Besides, the stability of the considered system at the equilibrium points was analyzed via numerical examples.

solar power satellite (SPS), Hamilton system, symplectic Runge-Kutta method, sail tower SPS

doi: 10.1360/N092016-00163 